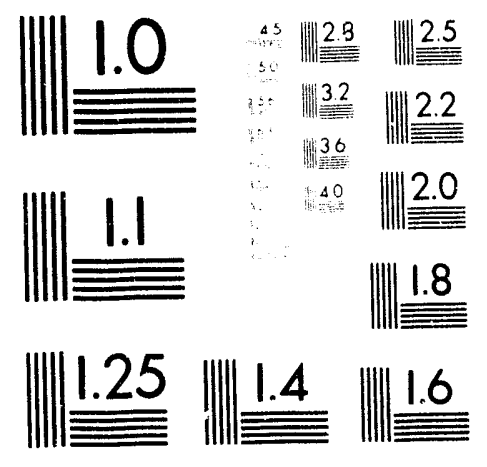



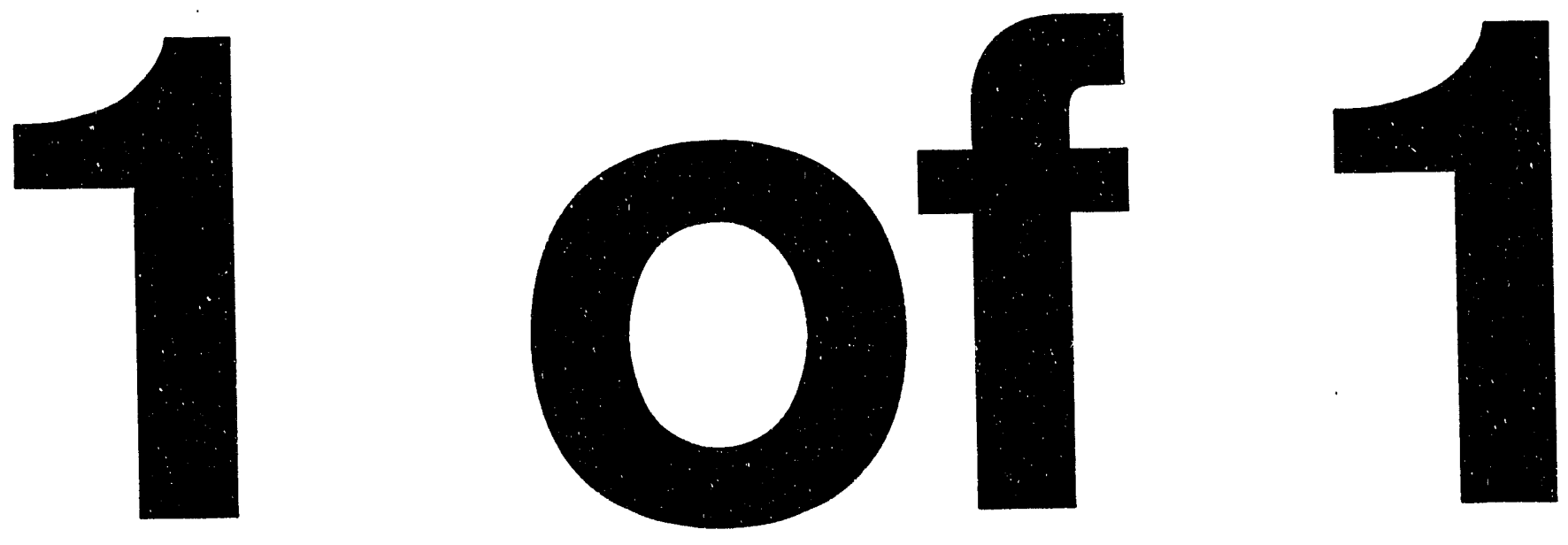


\title{
Sigma Matrix Reconstruction in the SLC Final Fucus*
}

\author{
P. RAIMAONDI, P. J. EMMA, N. TOGE, N. J. WALKER and V. ZIEMANN
}

Stanford Linear Accelerator Center, Stanford University, Stanford CA 94309

\section{Abstract}

The knowledge of the Beam Parameters at the entrance of the SLC final focus is important for modeling the final focus optics and predicting spot sizes and angular divergences at the interaction point (IP). It is also an important diagnostic for measuring possible anomalous emittance growth in the SLC Arcs. Reported here is a technique for measuring all the independent beam parameters at the beginning of the final focus. Use is made of the spot size measurements at wire scanners as a function of quadrupole strengths. Results for the SLC 1993 run are presented.

\section{INTRODUCTION}

The determination of all the independent beam parameters at the entrance of the SLC Final Focus has become a necessary task in order to understand anomalous emittances growth and beta-mismatch in the last two sections of the 50 $\mathrm{GeV}$ Linac and the Arcs. A full $4 \times 4$ sigma matrix reconstruction is required to determine the $X-Y$ coupling terms which arise from the Arcs. In addition to the coupling, anomalous emituance growth due to Synchrotron radiation and beam mismatch effects can be present [1], so a technique for measuring the uncoupled intrinsic emittances in the final focus is highly desirable.

\section{DESCRIPTION OF THE TECHNIQUE}

The sigma matrix reconstuction is performed by means of spot size measurements using a wire scanner (WS4) immediately upstream of the Superconducting final focus triplets.

This is normally a high "beta" point, but by retuning the beta matching section it is possible to bring both waists to the wire location and obtain spot sizes of the order of 100 um. Nexh, by varying two normal quads and one skew quad and measuring the $X, Y$ and $U$ ( $45^{\circ}$ wire) spots at the different quad settings, it is possible to extract all the beam parameters. Fig. 1 shows an example of the $\sigma_{x}{ }^{2}, \sigma_{y}{ }^{2}$ and $\sigma_{u}{ }^{2}$ as a function of a $x$-focusing quad.

*. Work supported by the Department of Energy,

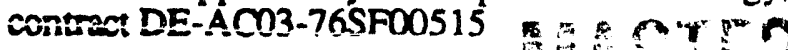

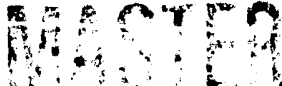

Presented at the Particle Accelerator Conference (PAC 93), Washington, DC, May 17-20, 1993
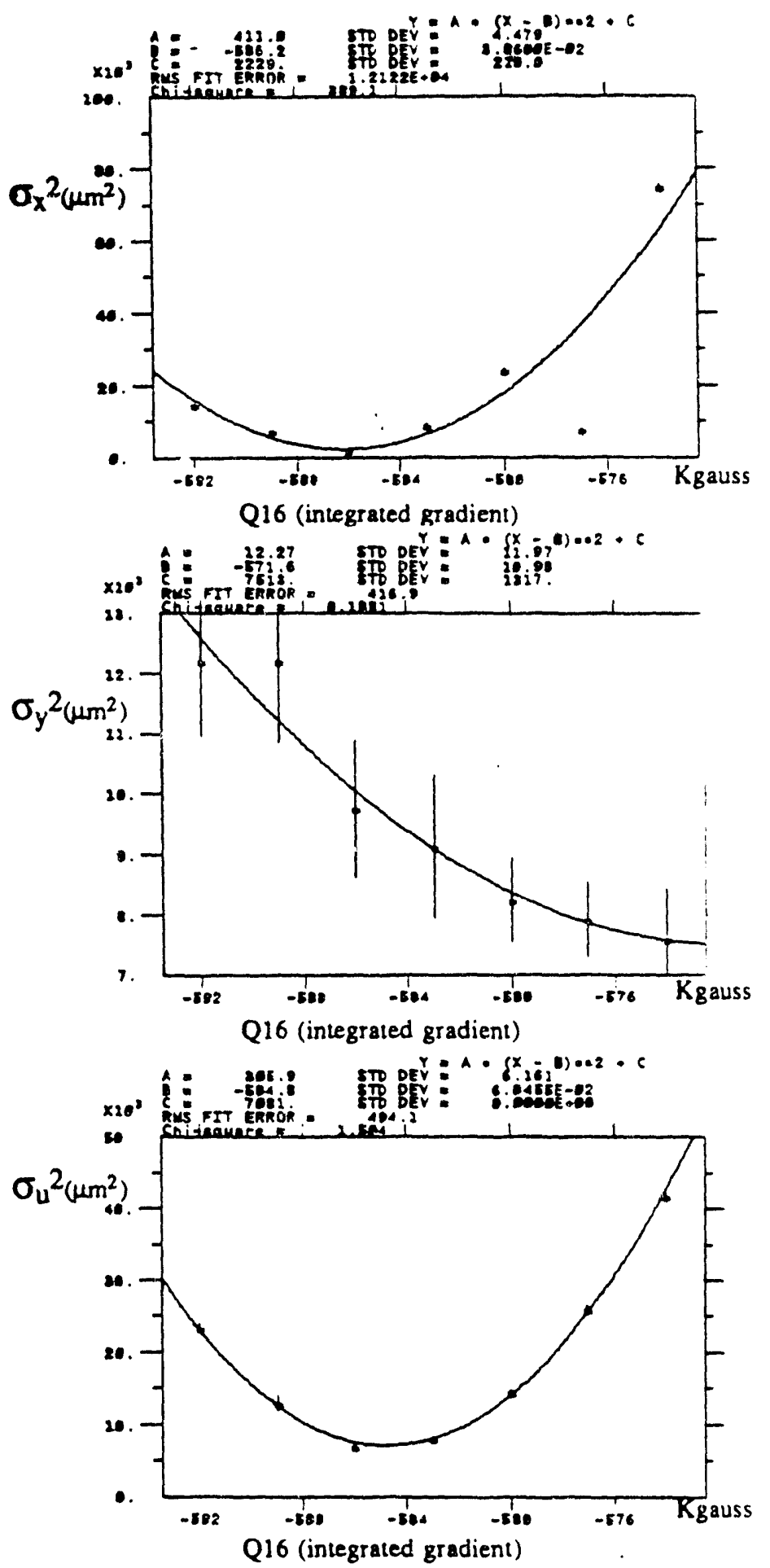

Figure 1. $X, Y$ and $U$ spol sizes as function of one of the measurement (Q16) quads.

¿k 
In an uncoupled treatment just the $X$ and $Y$ plots shown in Fig. 1 are sufficient to determine the beam twiss parameiers. With the addition of the coupling however, it is necessary to use three different quad scans and a non-linear fitting algorithm to extract the off-diagonal elements.

The $4 \times 4$ beam sigma covariance matrix $\sigma^{2}$ is parametrized as follows:

$$
\sigma^{2}=B \cdot C \cdot E \cdot C^{\prime} \cdot B^{\prime}
$$

where:

$$
\begin{aligned}
& E=\left[\begin{array}{llll}
e x & 0 & 0 & 0 \\
0 & \varepsilon x & 0 & 0 \\
0 & 0 & \varepsilon y & 0 \\
0 & 0 & 0 & \varepsilon y
\end{array}\right] \\
& C=\left[\begin{array}{cccc}
h & 0 & g \cdot c & g \cdot d \\
0 & h & -g \cdot a & -g \cdot b \\
g \cdot b & g \cdot d & h & 0 \\
-g \cdot a & -g \cdot c & 0 & h
\end{array}\right] \\
& B=\left[\begin{array}{cccc}
\sqrt{\beta_{x}} & \alpha_{x} \cdot \sqrt{\beta_{x}} & 0 & 0 \\
0 & \frac{1}{\sqrt{\beta_{x}}} & 0 & 0 \\
0 & 0 & \sqrt{\beta_{y}} & \alpha_{y} \cdot \sqrt{\beta_{y}} \\
0 & 0 & 0 & \frac{1}{\sqrt{\beta_{y}}}
\end{array}\right] \\
& g=\frac{\sin (\sqrt{\Delta})}{\sqrt{\Delta}}, h=\cos (\sqrt{\Delta}), \Delta=a \cdot d-c \cdot b
\end{aligned}
$$

The fitting is performed by minimizing the $\chi^{2}$ sum:

$$
\chi^{2}=\sum_{i=1}^{n}\left(\frac{\sigma_{i}^{\text {exp }}-\sigma_{i}^{\text {theo }}\left(\varepsilon_{x}, \beta_{x}, \alpha_{x}, \varepsilon_{y}, \beta_{y}, \alpha_{y}, a, b, c, d\right)}{\Delta \sigma_{i}^{\exp }}\right)^{2}
$$

where $\varepsilon_{x}, \beta_{x}, \alpha_{x}, \varepsilon_{y}, \beta_{y}, \alpha_{y}, a, b, c$ and $d$ are the fiuted parameters, and $\sigma_{i} \exp$ are the measured $X, Y$ and $U$ spot sizes.

\section{RESULTS}

Table 1 shows the results for two particular measurements of the positron $\sigma^{2}$ during the 1993 run. The first measurement was made with. the round beam configuration ( $\varepsilon_{x}-\varepsilon_{y}$ at the end of the Linac), the second one was made in the flat beam configuration $\left(\varepsilon_{x} \gg \varepsilon_{y}\right)$. The agreement with the predicted emittance growth from the Arcs is very good and the residual coupling is small.

Table 1

Measured positron beam parameters a the entrance of the final focus in the "round" and "flat" beam cases.

\begin{tabular}{|l|c|c|c|c|}
\cline { 2 - 5 } \multicolumn{1}{c|}{} & \multicolumn{2}{c|}{ Round beam } & \multicolumn{2}{c|}{ Flat beam } \\
\cline { 2 - 5 } \multicolumn{1}{c|}{} & Theoretic & Measured & Theoretic & Measured \\
\hline$\beta_{x}(\mathrm{ml})$ & 8.70 & $12.09 \pm 1.06$ & 8.70 & $6.99 \pm 0.69$ \\
\hline$\alpha_{x}$ & 0.00 & $1.09 \pm 0.16$ & 0.00 & $1.50 \pm 0.18$ \\
\hline$\varepsilon_{x}(\mathrm{E}-10)$ & 7.20 & $7.44 \pm 0.77$ & 9.60 & $10.46 \pm 1.23$ \\
\hline$\beta_{y}(\mathrm{mt})$ & 1.14 & $1.38 \pm 0.27$ & 1.14 & $1.44 \pm 0.48$ \\
\hline$\alpha_{y}$ & 0.00 & $0.10 \pm 0.18$ & 0.00 & $0.80 \pm 0.07$ \\
\hline$\varepsilon_{y}(\mathrm{E}-10)$ & 3.50 & $3.59 \pm 0.45$ & 0.65 & $0.60 \pm 0.12$ \\
\hline$a$ & 0.00 & $0.19 \pm 0.20$ & 0.00 & $0.32 \pm 0.17$ \\
\hline$b$ & 0.00 & $0.42 \pm .21$ & 0.00 & $0.41 \pm 0.13$ \\
\hline$c$ & 0.00 & $-0.30 \pm 0.15$ & 0.00 & $0.30 \pm 0.12$ \\
\hline$d$ & 0.00 & $-0.11 \pm 0.13$ & 0.00 & $0.50 \pm 0.12$ \\
\hline
\end{tabular}

Using these value we have been able to successfully predict the values for the beta-matching quads in order to cancel the residual coupling and to set to optimal beta functions at the interaction point .

\section{CONCLUSIONS}

The knowledge of the incoming sigma matrix in the final focus is a useful beam diagnostic and has been successful used in optimizing the first order final-focus optic during the 1992 and in the course of the 1993 SLC runs.

\section{REFERENCES}

[1] Phase Space and Synchrotron Radiation Emission in High Energy Electrons Transport lines. W.SPENCE, PEMMA, N.WALKER: IEEE-PAC93 proceedings. 

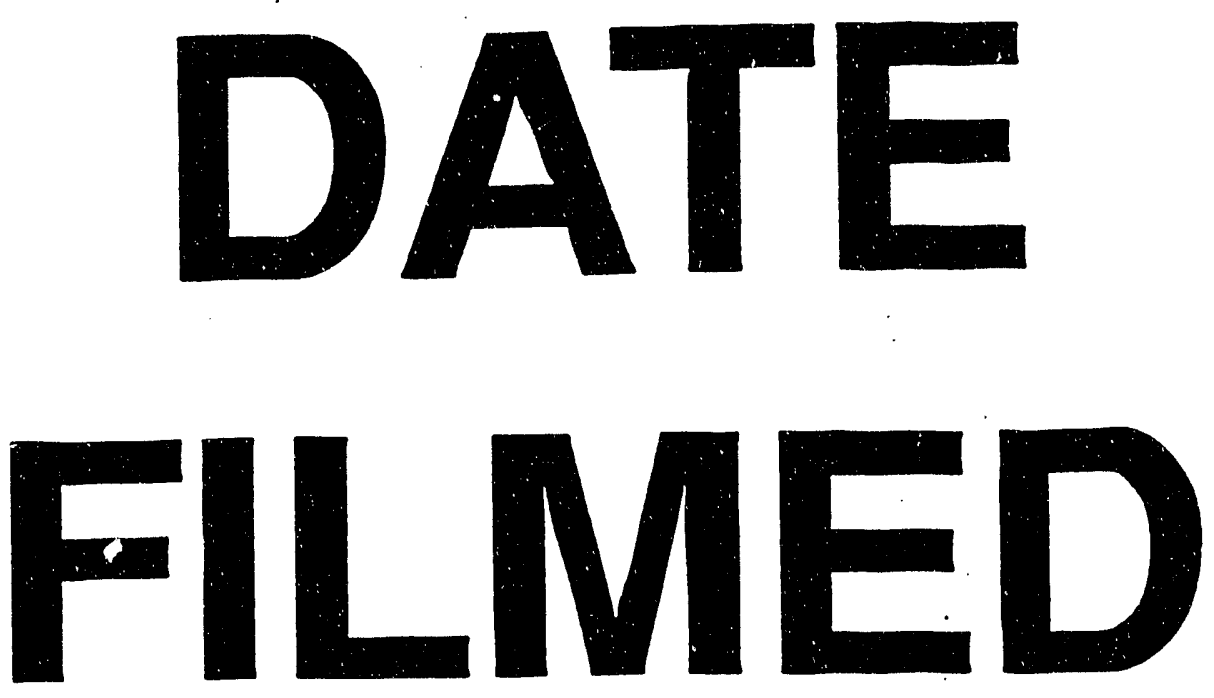

$11 / 9 / 93$
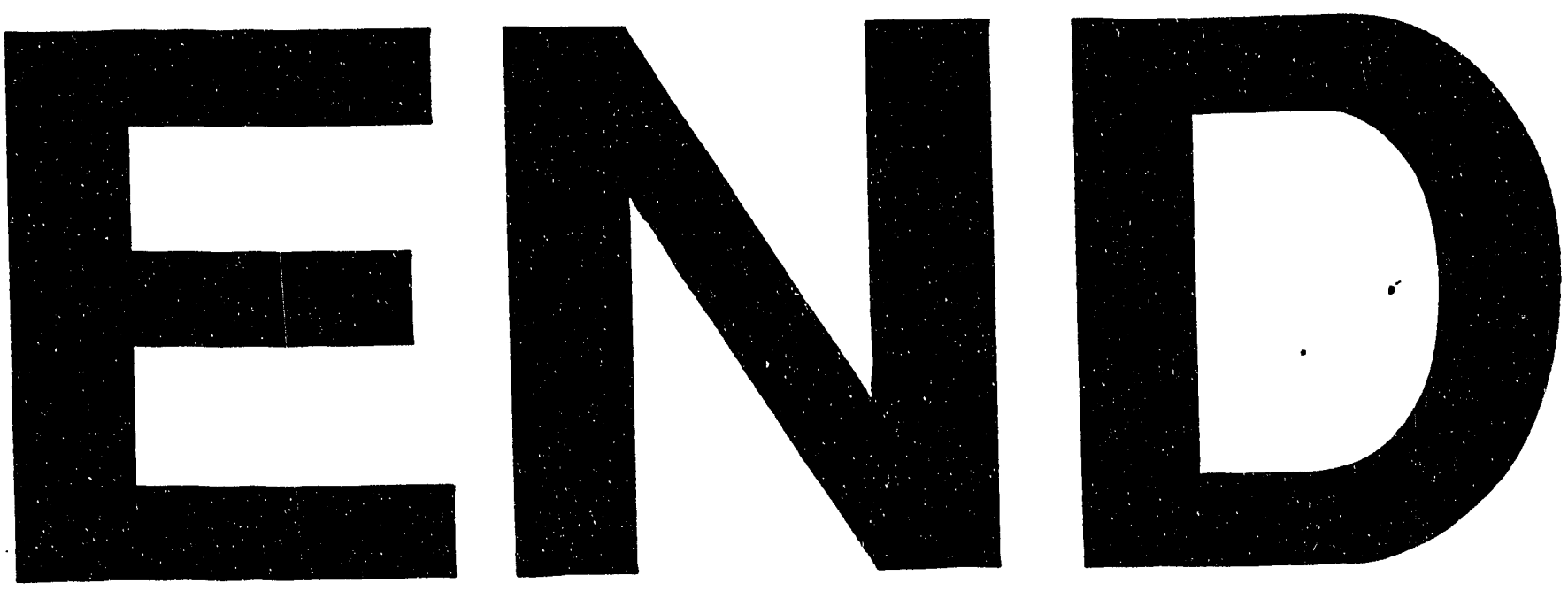
